\title{
MRONJ: due approcci chirurgici a confronto
}

\section{MRONJ: comparison between two surgical approaches}

\author{
Ricevuto il 17 dicembre 2019 \\ Accettato il 15 aprile 2020 \\ *Autore di riferimento \\ Emanuele Zanola \\ emanuelezanola1@gmail.com
}

Magda Mensi ${ }^{1}$

Elisabetta Audino

Eleonora Scotti

Emanuele Zanola ${ }^{1}$

Elisa Bettinsoli

Stefano Negrini ${ }^{2}$

Luca Visconti

Stefano Alessandro Salgarello 1
1Università di Brescia, Clinica

Odontoiatrica, Dipartimento di Specialità

Chirurgiche, Scienze Radiologiche

e Sanità Pubblica

¿U.O.C. Chirurgia Maxillo-Facciale,

Spedali Civili Brescia

\section{RIASSUNTO}

\section{OBIETTIVI}

Questo studio retrospettivo osservazionale ha come scopo quello di valutare la percentuale di recidive di MRONJ in pazienti onco-ematologici sottoposti a due differenti interventi chirurgici, uno meno invasivo (A) e uno più radicale (B).

Con MRONJ si intende un'"esposizione ossea con fistola intra- o extraorale nella regione mascellare, persistente per più di 8 settimane in pazienti che hanno o stanno assumendo farmaci correlati con ONJ, in assenza di pregressa terapia radiante". I farmaci associati al rischio di ONJ sono generalmente prescritti per trattare secondarismi ossei da tumore solido, lesioni osteolitiche da mieloma multiplo, ipercalcemia maligna o malattie osteometaboliche. Tali farmaci appartengono alla classe dei bifosfonati, agli anti-riassorbitivi contenenti anticorpi monoclonali e anti-angiogenetici.

Negli ultimi anni si è notata l'efficacia della prevenzione primaria della MRONJ, consistente nell'elimina- zione dei fattori locali di rischio nei pazienti a rischio ONJ ed effettuando trattamenti odontoiatrici prima di iniziare la terapia.

\section{MATERIALI E METODI}

Lo studio, relativo a 77 pazienti oncologici ed ematologici, è stato svolto a partire da dati raccolti presso I'U.O. Odontostomatologia e I'U.O. Chirurgia Maxillo-Facciale dell'ASST Spedali Civili di Brescia tra novembre 2016 e luglio 2018. Tutti i pazienti sono stati o sono ancora in terapia con bifosfonati o denosumab. I pazienti inclusi nello studio hanno un follow-up minimo di un anno.

I pazienti sono stati suddivisi in due gruppi a seconda del tipo di intervento chirurgico a cui sono stati sottoposti: interventi più conservativi (gruppo A) e interventi più radicali (gruppo B). II tipo di intervento chirurgico è stato scelto sulla base della stadiazione della patologia MRONJ; è stata utilizzata la stadiazione proposta dall'American Association of Maxillofacial Surgeons.
Entrambi i gruppi di pazienti sono omogenei dal punto di vista del protocollo pre- e post-chirurgico, nonché nel programma di followup. Si è scelto come termine per definire l'esito il momento di manifestazione della recidiva stessa.

Gli interventi chirurgici più conservativi si limitavano a curettage ossei 0 sequestrectomie in anestesia locale; gli interventi più radicali comprendevano chirurgia resettiva marginale 0 segmentale in anestesia generale.

\section{RISULTATI}

L'analisi statistica è stata condotta su un gruppo di 52 pazienti, in quanto di 25 pazienti dei 77 totali non è stato possibile risalire alla patologia di base 0 al tipo di intervento svolto.

L'analisi della percentuale di recidive negli interventi chirurgici porta a un P-value di 1, statisticamente non significativo, con un odds ratio di 1,09 . È stata analizzata la percentuale di recidive in interventi chirurgici meno invasivi (curettage ossei, sequestrectomie) rispetto alla per- centuale di recidive con procedure chirurgiche più radicali (chirurgia resettiva marginale o segmentale). Il numero di recidive nel gruppo $\mathrm{A}$ (interventi meno invasivi) è risultato essere di 4 casi su 24, mentre nel gruppo $B$ (intervento più invasivo) è risultato essere di 5 casi su 28.

\section{CONCLUSIONI}

I risultati dello studio non mostrano alcuna differenza statisticamente significativa tra i due approcci chirurgici. Dato il campione ristretto, sono necessari ulteriori studi, in particolare RCT.

\section{SIGNIFICATO CLINICO}

I risultati dello studio non mostrano alcuna differenza statisticamente significativa tra i due approcci chirurgici.

\section{PAROLE CHIAVE}
Osteonecrosi
- Bifosfonati
- Denosumab
Chirurgia
- Trattamento 


\section{ABSTRACT}

\section{OBJECTIVES}

The aim of this retrospective observational study is the comparison between two different surgical approaches for the MRONJ treatment in terms of healing and absence of recurrences. These different surgical techniques are divided into conservatives (group A) and radical (group B).

MRONJ means "exposed bone or bone that can be probed through an intraoral or extraoral fistula in the maxillofacial region that has persisted for longer than 8 weeks in patients who have or are taking ONJ-related drugs and in the absence of radiation therapy".

Drugs associated with ONJ development risk are used to treat bone metastases from solid cancers, osteolytic lesions from multiple myeloma, malignant hypercalcaemia or osteometabolic diseases. These drugs belong to bisphosphonates, antiresorptive and antiangiogenetics class.

In recent years, the efficacy of primary prevention of MRONJ has been noted. The primary prevention consists in the elimination of local risk factors and by carrying out dental treatments before starting therapy.

\section{MATERIALS AND METHODS}

The study analyses reports on 77 oncology and hematology patients. The data were collected from the Stomatology and MaxilIo-Facial Surgery Departments of ASST Spedali Civili of Brescia, from November 2016 to February 2018. The patients had taken or are taking bisphosphonate therapy or denosumab therapy. The patients included in the study have a minimum follow-up of one year.

The patients were divided into two groups according to the type of surgical approaches: the group A if the surgical technique is more conservative, group B if the surgical approaches is more radical. The type of surgical approaches is decided according with the MRONJ staging, proposed by American Association of Maxillofacial Surgeons.
The groups are homogeneous for the pre- and post-surgical protocols and for the follow-up program. The outcome was defined with the recurrence.

The conservative surgical approaches were limited to bone curettage or sequestrectomies under local anesthesia; the radical surgical approaches included marginal or segmental resective surgery under general anesthesia.

\section{RESULTS}

The statistical analysis was conducted on a group of 52 patients, since 25 of the 77 initial patients could not be traced back to the underlying pathology or type of surgical technique performed.

Analysis of recurrence rates in surgical procedures show that are not statistically significant difference $(P$-value $=1$; odds ratio $=1.09)$.

The percentage of recurrences in conservative surgical approaches (bone curettage, sequestrectomies) was analyzed compared to the percentage of recurrences in radical surgical procedures (mar- ginal or segmental resective surgery). The number of recurrences in group $A$ (conservative surgical approaches) was 4 out of 24 cases, while in group $B$ (radical surgical approaches) it was 5 out of 28 cases.

\section{CONCLUSIONS}

The results of the study show that there is no statistically significant difference between minimally invasive and invasive surgery technique. The results could be influenced by the small sample size, so further studies are needed, in particular RCT.

\section{CLINICAL SIGNIFICANCE}

The results of the study show that there is no statistically significant difference between minimally invasive and invasive surgery technique.

\section{KEY WORDS}
Osteonecrosis
- Bisphosphonates
Denosumab
- Surgery
- Treatment

\section{INTRODUZIONE}

L'osteonecrosi, per definizione, è la morte del tessuto osseo dovuta a un insufficiente apporto sanguigno. Quando questa interessa le ossa mascellari e mandibolari prende il nome di Osteonecrosis of the Jaw (ONJ) e, a seconda della causa primaria scatenante, assume diverse terminologie. In questo studio prenderemo in esame l'osteonecrosi dei mascellari correlata all'assunzione di specifici farmaci.
L'American Association of Oral and Maxillofacial Surgeons (AAOMS) ha fissato la definizione di MRONJ (Medication-Related Osteonecrosis of the Jaws) come "esposizione ossea od osso che può essere esaminato attraverso una fistola intra- o extra-orale nella regione mascellare, persistente per più di 8 settimane in pazienti che hanno o stanno assumendo farmaci correlati con ONJ"[1].

Più in generale, si può definire MRONJ come una patologia consistente nella progressiva distruzione ossea mascellare/mandibolare a seguito di trattamento con farmaci correlati al rischio di $\mathrm{ONJ}$, in assenza di un precedente trattamento radiante ${ }^{[2]}$.

I farmaci associati al rischio di sviluppo di ONJ sono generalmente appartenenti alla classe dei bifosfonati (per esempio, zolendronato, pamidronato, alendronato), agli anti-riassorbitivi composti da anticorpi monoclonali (per esempio, denosumab) e agli anti-angiogenetici (per esem- 
pio, sutent e subitinib). Tali farmaci vengono prescritti a pazienti con specifiche patologie di base, per esempio lesioni osteolitiche da mieloma multiplo, secondarismi ossei da tumore solido primitivo, ipercalcemia maligna o malattie osteometaboliche (osteoporosi, morbo di Paget, artrite reumatoide).

Il primo caso di osteonecrosi correlata a un farmaco bifosfonato fu descritto da Marx nel 2003 ${ }^{[3]}$, ma da allora l'esatta eziopatogenesi non è ancora stata scoperta. Tuttavia l'osteonecrosi dei mascellari è un fenomeno avverso che, pur considerato raro, se non trattato precocemente e correttamente può avere conseguenze debilitanti per il paziente fino a comprometterne gravemente la qualità di vita.

Con la descrizione di Marx nel 2003 si definì la BRONJ (Bisphosphonate-Related Osteonecrosis of the Jaws); nel corso degli anni si è notata la correlazione anche con altre categorie di farmaci: prima gli anti-riassorbitivi da cui si è definita l'ARONJ (Anti-resorptive Related Osteonecrosis of the Jaws) e, infine, gli anti-angiogenetici da cui si è stabilita la più generica definizione di MRONJ.

Tuttavia, i termini BRONJ, ARONJ e MRONJ vengono ancora interscambiati tra loro nella letteratura, creando non poca confusione nella ricerca bibliografica di dati univoci per quanto riguarda incidenza e prevalenza; inoltre, spesso tale fenomeno viene poco segnalato ai centri di riferimento per la farmacovigilanza. L'insieme di questi fattori ha fatto sì che la letteratura presenti studi con risultati non sempre coerenti tra loro. Analizzando ciò che è presente in letteratura per quanto concerne la frequenza del manifestarsi di ONJ, i pazienti vengono suddivisi in due categorie a seconda della patologia di base per cui assu- mono i farmaci suddetti: pazienti oncologici e pazienti non oncologici.

Per quanto riguarda il rischio complessivo, la letteratura riporta una percentuale che varia dallo 0 al 6,7, con una media negli studi compresa tra l' $1 \%$ e il $3 \%$ per i pazienti oncologici; il rischio scende nei pazienti non oncologici in un intervallo dallo $0,004 \%$ allo $0,2 \%$, con una media negli studi che si aggira intorno allo $0,1 \%{ }^{[4]}$.

In una review sistematica della letteratura del 2014 di Khan e colleghi, si legge che per quanto riguarda la prevalenza di ONJ nei pazienti osteoporotici assumenti bifosfonati l'intervallo è compreso tra lo $0 \%$ e lo $0,04 \%$, mentre l'incidenza varia da 1,04 a 69 per 100.000 pazienti/ anno. L'incidenza di ONJ nella stessa categoria di pazienti, ma assumenti denosumab, varia da 0 a 30,2 per 100.000 pazienti/anno ${ }^{[5]}$.

Per quanto riguarda i pazienti oncologici, Khan e colleghi scrivono che la prevalenza di ONJ si aggira tra lo $0 \%$ e lo $0,186 \%$, mentre l'incidenza ha un intervallo compreso tra 0 e 12.222 per 100.000 pazienti/anno nei soggetti assumenti bifosfonati. Per quanto riguarda i pazienti assumenti denosumab il range va da 0 a 2316 per 100.000 pazienti/anno ${ }^{[5]}$.

L'incidenza di ONJ nella popolazione oncologica comprende variabili confondenti, per esempio altri farmaci che influiscono sulla salute ossea quali glucorticoidi e farmaci anti-angiogenetici. Secondo Christodoulou e colleghi, la prevalenza di ONJ cresce fino al $16 \%$ se i bifosfonati sono assunti in combinazione con gli anti-angiogenetici[i].

Negli ultimi anni si è notata l'efficacia della prevenzione primaria della MRONJ, consistente nell'eliminazione dei fattori locali di rischio nei pazienti a rischio ONJ (presenza di eventuali foci infettivi, problemi parodontali e/o perimplantari) e pianificando gli eventuali trattamenti odontoiatrici prima di iniziare la terapia con i farmaci correlati all'ONJ. Nonostante la chiara efficacia della prevenzione primaria, permangono casi $\mathrm{d}$ fenomeni avversi con diagnosi di ONJ, i quali vengono trattati chirurgicamente.

Gli obiettivi del trattamento dei pazienti con una diagnosi certa di MRONJ sono l'eliminazione del dolore, il controllo dell'infezione dei tessuti molli e duri e la riduzione al minimo della progressione della patologia. I pazienti con MRONJ riconosciuta dovrebbero evitare procedure chirurgiche dento-alveolari elettive; infatti, questi siti chirurgici potrebbero comportare ulteriori aree di osso necrotico esposto.

Dalla pubblicazione delle linee guida nel 2009 , sono stati segnalati diversi casi di successo con terapia chirurgica (sequestrectomia, resezione ossea) in tutti gli stage di MRONJ ${ }^{[7,8]}$.

Le aree di osso necrotico sono una costante fonte di lesione per i tessuti molli, indipendentemente dallo stadio della malattia. Per questo motivo i sequestri ossei andrebbero rimossi quanto prima e l'osso sano rimanente dovrebbe essere modificato in maniera da non causare ulteriori problemi ai tessuti circostanti, garantendo la guarigione ${ }^{[0]}$. È da considerare anche l'eventuale estrazione di denti sintomatici nella compagine ossea necrotica, dato che sembra improbabile che l'estrazione possa esacerbare il processo necrotico stabilito.

Sono presenti in letteratura segnalazioni di casi con piccoli campioni documentati in cui vengono usate altre strategie di trattamento non chirurgico, come plasma ricco di piastrine, irraggiamento laser a basso livello[9,10], ormone 
paratiroideo ${ }^{[11]}$ e proteina ossea morfogenica. L'efficacia di queste terapie e le modalità di trattamento devono essere stabilite attraverso ulteriori ricerche $e$ studi controllati.

L'obiettivo di questo studio è quello di valutare la percentuale di recidive di MRONJ in pazienti onco-ematologici affetti da MRONJ e sottoposti a due differenti tipi di interventi chirurgici, uno $(A)$ meno invasivo e uno $(B)$ più radicale.

\section{MATERIALI E METODI Disegno dello studio}

Quello presentato è uno studio retrospettivo osservazionale svolto sui dati di pazienti oncologici ed ematologici che sono stati visitati e trattati presso l'U.O. Clinica Odontoiatrica e presso I'U.O. Chirurgia Maxillo-Facciale dell'ASST Spedali Civili di Brescia da novembre 2006 a luglio 2018.

Non è stata necessaria l'approvazione da parte del Comitato Etico per tale tipologia di studio.

\section{Pazienti e farmaci}

Sono stati considerati gli interventi eseguiti fino a luglio 2018. I pazienti sono stati trattati presso l'ASST Spedali Civili di Brescia, in particolare nei reparti di U.O. Chirurgia Maxillo-Facciale e di U.O. Clinica Odontoiatrica e, sulla base della gravità della patologia $\mathrm{ONJ}$, si sono svolti interventi più o meno radicali $e$ in anestesia generale o locale.

Ogni paziente di ciascun gruppo ha seguito un programma minimo di followup post-chirurgico composto da controlli a 1 settimana, a 1 mese, a 6 mesi e a un anno. Si è scelto come termine per definire l'esito il momento di manifestazione della recidiva stessa, che si è manifestata a distanza nel gruppo $\mathrm{A}$ da un minimo di 4 mesi fino a un massimo di 5 anni, mentre nel gruppo $B$ da 3 mesi a 1 anno dall'intervento.

Per "recidiva" si intende l'interruzione della continuità della mucosa orale con esposizione dell'osso mandibolare/mascellare sottostante oppure la comparsa di fistole intra-orali nel sito in cui è avvenuto l'intervento chirurgico di rimozione dell'osso necrotico. Tale osso, in seguito all'esposizione, è andato poi incontro a infezione e a successiva necrosi.

Per determinare lo stadio della patologia è stata utilizzata la stadiazione proposta dall'AAOMS ${ }^{[1]}$ :

- soggetto a rischio, paziente che non presenta esposizione di osso necrotico e che non ha sintomi, ma che assume bifosfonati per os o ev;

- stage 0 , paziente che assume bifosfonati o farmaci correlati, senza esposizione di osso necrotico nel cavo orale, ma che presenta caratteristiche cliniche non specifiche (per esempio, flogosi), cambiamenti riscontrabili a livello radiografico o sintomi;

- stage 1, paziente che assume bifosfonati o farmaci correlati che presenta esposizione di osso necrotico a livello del cavo orale o fistola mucosa attraverso la quale è possibile fare un sondaggio fino all'osso sottostante. ॥ paziente risulta però essere asintomatico e non vi è l'evidenza di infezione;

- stage 2, paziente che assume bifosfonati o farmaci correlati che presenta esposizione di osso necrotico a livello del cavo orale o fistola mucosa attraverso la quale è possibile fare un sondaggio fino all'osso sottostante. In questo caso l'esposizione ossea è associata a infezione che comporta dolore ed eritema nella zona di osso esposto. Può esserci oppure no anche essudato purulento;
- stage 3, paziente che assume bifosfonati o farmaci correlati che presenta esposizione di osso necrotico a livello del cavo orale con dolore, infezione e almeno una delle seguenti condizioni: osso esposto e necrotico che si estende oltre l'osso alveolare comportando fratture patologiche, fistola extra-orale, sviluppo di comunicazioni oro-antrali od oro-nasali, osteolisi che si estende fino al bordo inferiore della mandibola o al pavimento del seno mascellare.

\section{Criteri di selezione}

I pazienti ritenuti validi per il presente studio dovevano avere le seguenti caratteristiche:

- essere pazienti oncologici;

- assumere/aver assunto farmaci antiriassorbitivi;

- aver avuto diagnosi di ONJ;

- essere stati sottoposti a intervento chirurgico per la rimozione dell'ONJ;

- avere un follow-up di almeno 1 anno.

Sono invece stati esclusi dal presente studio:

- pazienti oncologici che assumevano/ avevano assunto farmaci antiriassorbitivi, ma le cui condizioni di base non hanno consentito un intervento chirurgico (per esempio, pazienti osteoporotici, malattia di Paget);

- pazienti affetti da altre patologie osteometaboliche che assumevano/avevano assunto farmaci antiriassorbitivi;

- pazienti in stato di gravidanza.

\section{Procedure chirurgiche}

I pazienti dello studio sono stati visitati o presso I'U.O. Clinica Odontoiatrica o presso I'U.O. Chirurgia Maxillo-Facciale dell'ASST Spedali Civili di Brescia. Dopo avere preso visione di esami radiografici (ortopantomografia e/o TC Cone Beam), 
a seconda della gravità della patologia, i pazienti sono stati indirizzati per la procedura chirurgica o presso I'U.O. Clinica Odontoiatrica o presso I'U.O. Chirurgia Maxillo-Facciale dell'ASST Spedali Civili di Brescia: gli stadi 0 e 1 sono stati trattati in anestesia locale anche presso la prima U.O., mentre gli stadi 3 e 4 hanno necessitato dell'anestesia generale.

Prima e dopo ogni intervento i pazienti sono stati sottoposti a terapia antibiotica con amoxicillina+acido clavulanico $(1 \mathrm{~g}$ ogni $8 \mathrm{~h}$ per 8 giorni prima dell'intervento e $1 \mathrm{~g}$ ogni $12 \mathrm{~h}$ per 8 giorni) e metronidazolo ( $250 \mathrm{mg}$ ogni $6 \mathrm{~h}$ per $15 \mathrm{gg}$ ). Ogni intervento è stato svolto solamente qualora le condizioni sistemiche del paziente lo consentissero.

Per quanto riguarda gli stadi 0 e 1 l'intervento, non essendo invasivo, ha previsto curettage ossei o sequestrectomie, mentre agli stadi 2 e 3 si sono svolti interventi più radicali, eseguiti con chirurgia resettiva marginale o segmentale. Questi ultimi due tipi di interventi prevedono l'incisione e lo scollamento di un lembo a tutto spessore che comprenda sia la componente ossea patologica sia quella sana. II segmento osseo su cui intervenire viene definito tramite esami sia clinici che radiologici, attraverso i quali possono essere visualizzate le strutture anatomiche coinvolte.

Gli interventi più conservativi vengono svolti in anestesia locale o in sedazione cosciente, mentre nei casi di chirurgia resettiva si opera in regime di anestesia generale.

\section{Curettage ossei}

Per curettage ossei o debridement si intende l'asportazione chirurgica del tessuto osseo necrotico fino all'identificazione di una superficie ossea sanguinante, seguita dalla chiusura completa del difetto mediante mobilizzazione di un lembo muco-periosteo. L'osso è asportato per strati mediante l'uso di frese rotanti o di strumenti a minore invasività (piezoelettrici). Questo intervento prevede che l'osso necrotico non sia ancora separato dall'osso vitale circostante (nel qual caso si parlerebbe di sequestrectomia). L'intervento di curettage dento-alveolare prevede l'aggiunta di un'osteoplastica della superficie ossea per eliminare eventuali asperità residue e consentire una chiusura per prima intenzione del lembo.

\section{Sequestrectomia}

II sequestro osseo consiste in una porzione di osso necrotico, includente o meno anche elementi dentari, che si distacca progressivamente dall'osso circostante che invece mantiene caratteristiche di vitalità. II sequestro osseo è un evento frequente nei processi osteonecrotici e osteomielitici. II processo di sequestrazione può portare all'espulsione spontanea del sequestro quando la porzione necrotica è separata completamente dall'osso vitale circostante me- diante interposizione di tessuto di granulazione ben vascolarizzato. In queste condizioni l'eliminazione del sequestro avviene senza la necessità di anestesia o di intervento chirurgico. Con il termine sequestrectomia si intende pertanto la rimozione chirurgica, eseguita in anestesia locale o generale, di un sequestro osseo generalmente interessante il processo alveolare dei mascellari e ancora solidale ai tessuti circostanti (figg. 1-4). Questo intervento è svolto in anestesia loco-regionale, generale o narcosi in relazione all'estensione del processo e alle condizioni cliniche del paziente. L'intervento di sequestrectomia prevede sempre in aggiunta un'osteoplastica della superficie ossea adiacente per eliminare possibili asperità residue e garantire una chiusura per prima intenzione del lembo mucoperiosteo.

\section{Chirurgia resettiva}

Con il termine di chirurgia resettiva si intende l'asportazione in blocco dell'osso patologico sino ad arrivare a tessuto verosimilmente sano. L'intervento resettivo nelle MRONJ deve sempre prevedere in

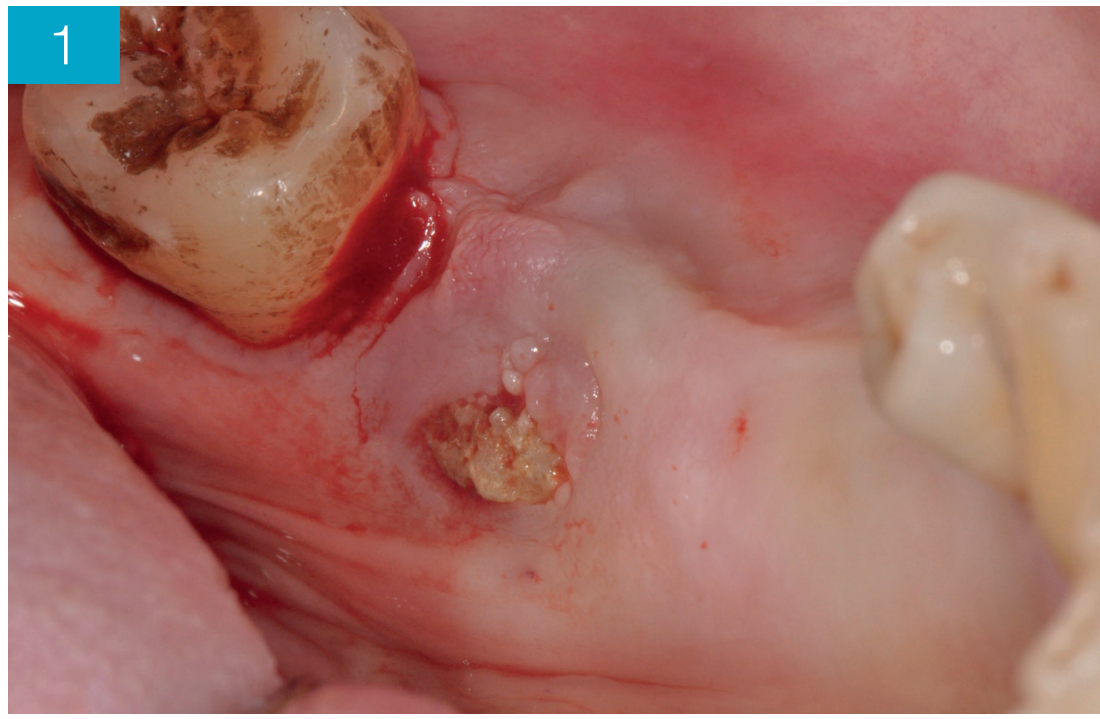

Fig. 1 Caso 1: foto pre-operatoria sede mandibolare ONJ 


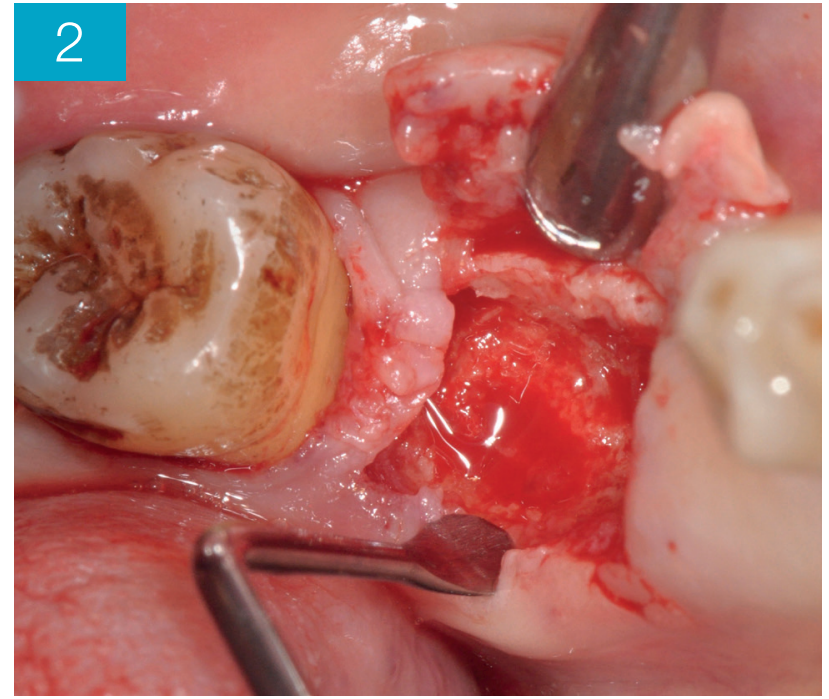

Fig. 2 Caso 1: foto intra-operatoria sede mandibolare ONJ

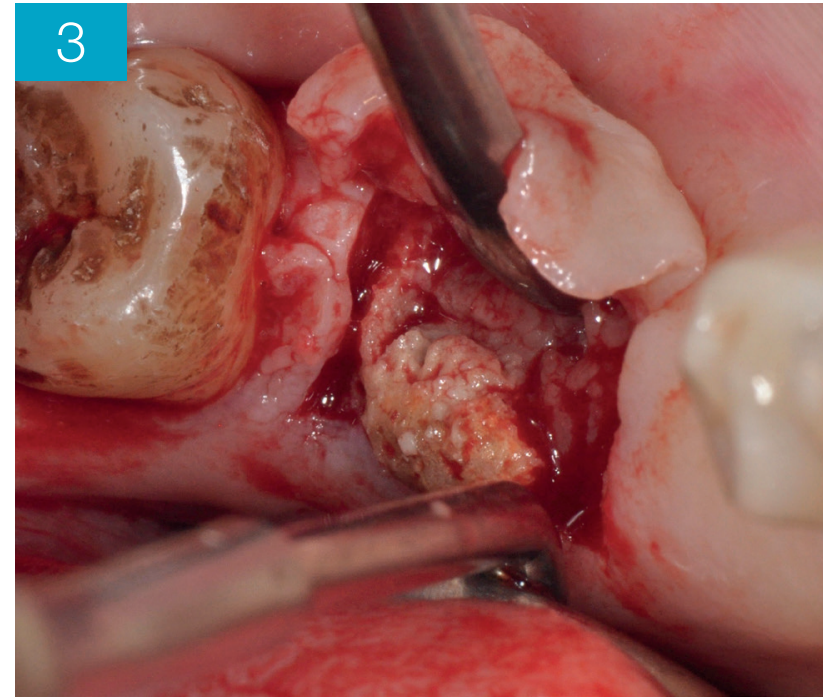

Fig. 3 Caso 1: esposizione osso necrotico aggiunta anche un'osteoplastica dei margini di resezione al fine di eliminare possibili asperità residue e assicurare la chiusura per prima intenzione del difetto mediante mobilizzazione di un lembo muco-periosteo. Diversamente dalla chirurgia oncologica, i margini di resezione ossea di MRONJ non sono codificati. Per definizione, un margine di resezione dovrebbe cadere nell'area di tessuto sano da un punto di vista clinico e radiografi-

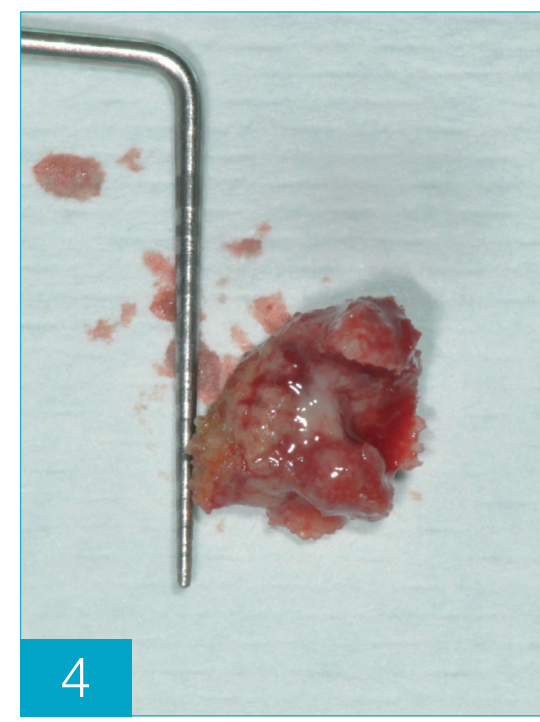

Fig. 4 Caso 1: osso necrotico rimosso co, adiacente al tratto di osso patologico da asportare, tale da garantire una guarigione stabile nel tempo.

Si distinguono due forme di chirurgia resettiva:

- chirurgia resettiva marginale, che consiste nell'asportazione in blocco del tessuto patologico, senza interruzione della continuità anatomica del segmento scheletrico interessato. L'intervento viene generalmente eseguito in anestesia generale previa ospedalizzazione. La resezione marginale interessa di regola i processi dento-alveolari della mandibola e del mascellare, limitandosi alle sole basi scheletriche in rare circostanze. A livello mandibolare, il canale osseo del fascio vascolo-nervoso alveolare inferiore rappresenta il limite naturale di resezione dentoalveolare. Al contrario, nel mascellare superiore la distinzione tra chirurgia marginale e segmentale è meno netta, poiché l'asportazione del processo dento-alveolare comporta tipicamente la creazione di una comunicazione oro-nasale e/o oro-antrale e quindi di una discontinuità ossea;
- chirurgia resettiva segmentale, che consiste nell'asportazione in blocco (a tutto spessore) di un segmento scheletrico con interruzione della sua continuità anatomica (figg. 5-8). L'intervento in questo caso è eseguito in anestesia generale e in regime di ospedalizzazione. Anche in questo caso è necessaria l'aggiunta di un'osteoplastica dei margini di resezione per eliminare possibili asperità residue $\mathrm{e}$ garantire la chiusura mucosa per prima intenzione del difetto mediante mobilizzazione di un lembo muco-periosteo, ove non indicata altra ricostruzione. Per quanto riguarda la mandibola, l'intervento standard è la mandibulectomia. Essa causa sempre la perdita della simmetria del terzo inferiore del volto e dell'occlusione. Per questo motivo è ritenuto opportuno ricostruire immediatamente la continuità ossea e ripristinare la morfologia del volto, favorendo un recupero funzionale ed estetico della zona interessata. La ricostruzione dell'integrità mandibolare dopo questo tipo di intervento può essere ottenuta mediante placche in titanio o con lembi ossei vascolarizzati. Per quanto riguarda invece l'intervento reset- 


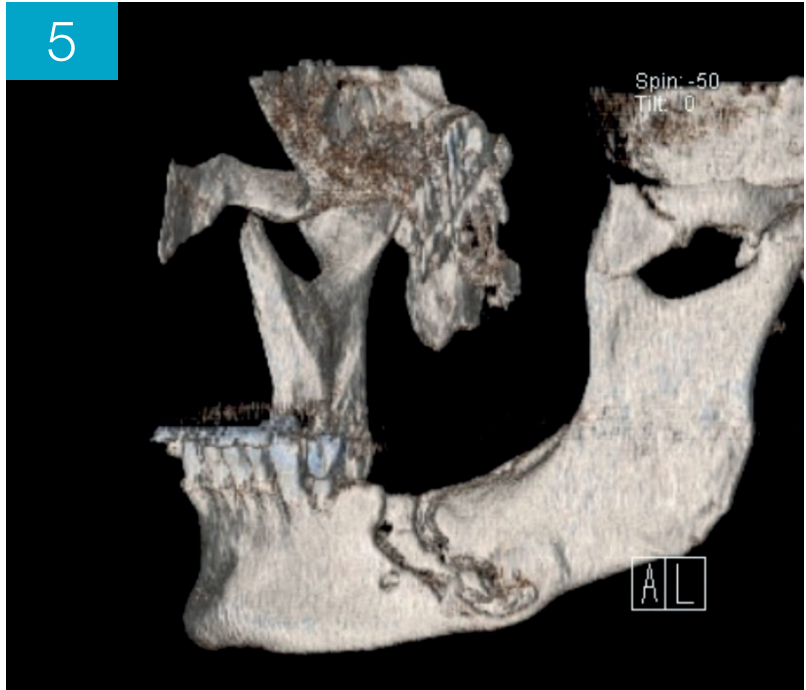

Fig. 5 Caso 2: ricostruzione 3D del difetto osseo

tivo segmentale per il mascellare superiore si parla di maxillectomia. Si distinguono maxillectomie parziali e totali, le prime caratterizzate dall'asportazione del solo processo alveolare, con o senza la conservazione del palato; le seconde sono contraddistinte invece dall'asportazione in blocco di tutte le pareti, incluso il pavimento orbitario.

Nel caso dello studio qui presentato, $\mathrm{i}$ pazienti che hanno subito l'intervento di curettage osseo o di sequestrectomia sono stati inseriti nel gruppo A, mentre i pazienti che sono stati sottoposti a intervento di chirurgia resettiva sono stati raggruppati nel gruppo $\mathrm{B}$.

\section{ANALISI STATISTICA}

L'analisi statistica è stata condotta su un gruppo di 52 pazienti, in quanto di 25 pazienti dei 77 totali non è stato possibile risalire alla patologia di base o al tipo di intervento subito.

L'analisi statistica è stata portata a termine mediante Test di Fisher con un intervallo di confidenza $(0,20 ; 6,26)$.
Fig. 7 Caso 2: segmento osseo patologico asportato

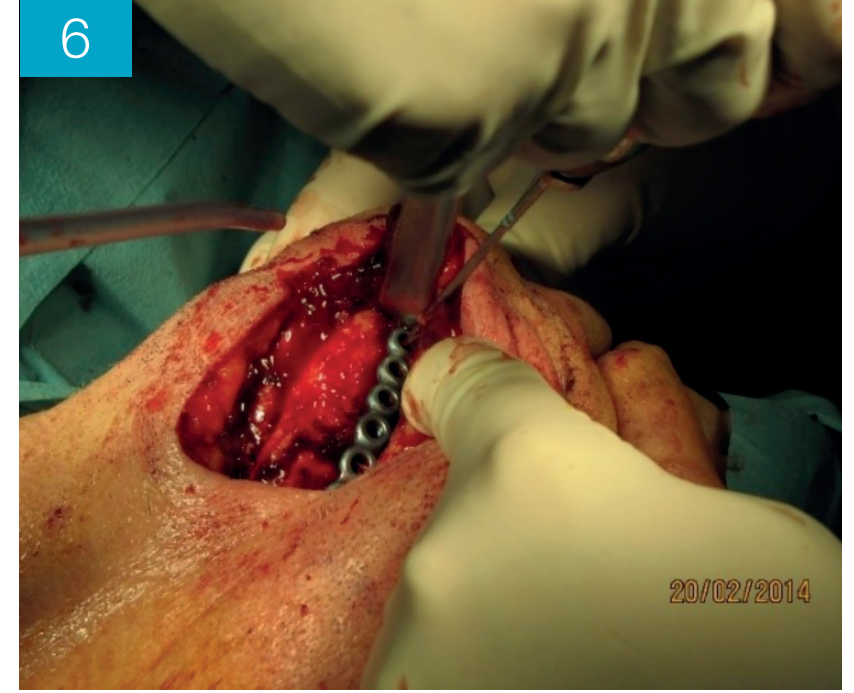

Fig. 6 Caso 2: posizionamento placca in titanio
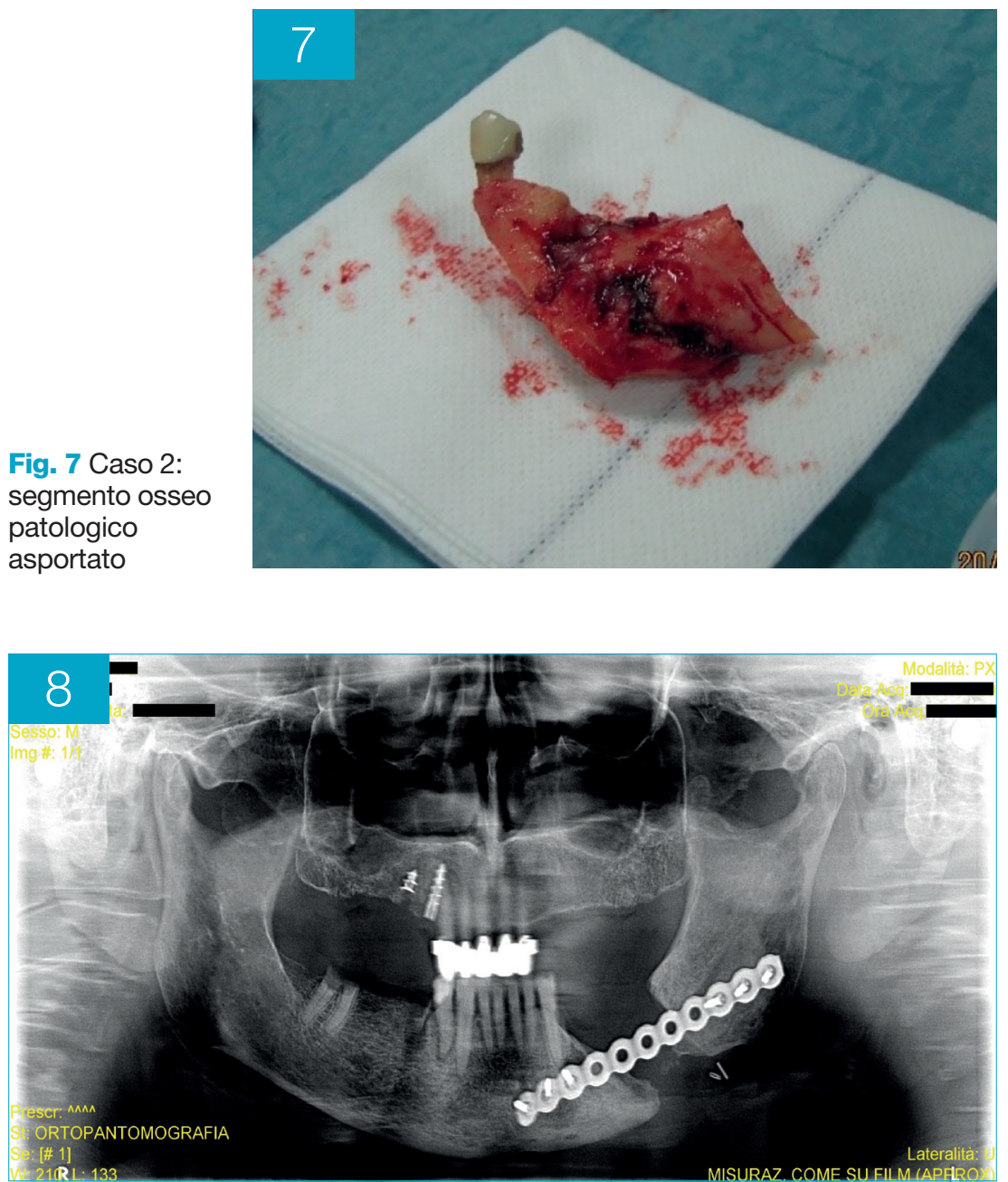

Fig. 8 Caso 2: ortopantomografia di controllo a 3 anni 


\section{RISULTATI}

I 77 soggetti che sono rientrati nello studio sono pazienti oncologici o ematologici che sono stati o sono ancora in terapia con bifosfonati o denosumab e che hanno avuto almeno un episodio di ONJ e di cui è stato possibile recuperare dati riguardanti patologia di base, farmaci assunti e data dell'evento avverso. Di questi pazienti, 52 sono di sesso femminile e 25 di sesso maschile. L'analisi statistica è stata condotta su un gruppo di 52 pazienti, in quanto di 25 pazienti dei 77 totali non è stato possibile risalire alla patologia di base 0 al tipo di intervento subito.

La maggior parte dei pazienti considerati in questo studio ha assunto o assume acido zoledronico (51), mentre gli altri assumono $\mathrm{o}$ altri bifosfonati o altri farmaci (1 risendronato, 8 pamidronato, 3 alendronato, 1 clodronato, 1 denosumab, 12 non specificato) (tab. I).

Per quanto riguarda la patologia di base per la quale è stata instaurata la terapia con BP o denosumab si hanno 42 pazienti affetti da tumore solido e 29 da mieloma multiplo. Per i restanti 6 pazienti non è stato possibile individuare l'esatta patologia di base (tab. II).

Tra i tumori solidi, quello maggiormente rappresentato è il Ca mammario (29), mentre sono presenti anche Ca prostatico (7), Ca renale (3), Ca orale (1), Ca parotideo (1), linfoma $\mathrm{NH}$ (1).

È stata analizzata la percentuale di recidive in interventi chirurgici meno invasivi (curettage ossei, sequestrectomie) rispetto alla percentuale di recidive con procedure chirurgiche più radicali (chirurgia resettiva marginale o segmentale). II numero di recidive nel gruppo A (interventi meno invasivi) è risultato essere di 4 casi su 24, mentre nel gruppo B (intervento più invasivo) è risultato essere di 5

\begin{tabular}{|c|c|}
\hline Tab. I Farmaci antiriassorbitivi assunti \\
\hline Farmaco assunto & Numero pazienti \\
\hline Zoledronato & 51 \\
\hline Pamidronato & 8 \\
\hline Alendronato & 3 \\
\hline Risendronato & 1 \\
\hline Clodronato & 1 \\
\hline Denosumab & 1 \\
\hline Non specificato & 1 \\
\hline Totale & 77 \\
\hline
\end{tabular}

\begin{tabular}{|c|c|}
\hline \multicolumn{2}{|c|}{ Tab. II Patologia di base } \\
\hline Patologia & Numero pazienti \\
\hline Mieloma multiplo & 29 \\
\hline Tumori solidi & 42 \\
\hline Ca mammario & 29 \\
\hline Ca prostatico & 7 \\
\hline Ca renale & 3 \\
\hline Ca orale & 1 \\
\hline Ca parotideo & 1 \\
\hline Linfoma NH & 1 \\
\hline Non specificato & 6 \\
\hline Totale & 77 \\
\hline
\end{tabular}

casi su 28. II P-value risulta essere di 1 e quindi statisticamente non significativo, con un odds ratio di 1,09.

\section{DISCUSSIONE}

Lo scopo di questo studio è stato quello di cercare di trovare una correlazione tra la tipologia di intervento e il rischio di recidive in pazienti oncologici ed ematologici affetti da MRONJ.

In uno studio di coorte di Ristow et. al. ${ }^{[12]}$ condotto su 75 pazienti con un totale di 92 siti affetti da MRONJ, trattati con interventi di tipo conservativo, solo 8 siti hanno ottenuto una completa guarigione. I restanti 84 hanno mantenuto l'esposizione ossea e 67 di questi hanno sviluppato un peggioramento della lesione.
In uno studio di Bodem et al. ${ }^{[13]}$ è stato dimostrato come interventi di tipo radicale in pazienti affetti da MRONJ di stadio $2 \mathrm{e}$ 3 risultassero nella completa guarigione del sito nel 75,5\% dei casi (35 su 47). La review sistematica di Comas-Calonge et al. ${ }^{[14]}$ ha sottolineato come l'intervento di tipo conservativo (sequestrectomia, osteotomia o curettage osseo) garantisse una guarigione del sito infetto con una percentuale variabile tra il 58 e il 100.

In uno studio Bedogni et al. ${ }^{[15]}$ hanno dimostrato come la chirurgia resettiva fosse utile nel trattare casi di MRONJ in pazienti onco-ematologici.

Nello studio che abbiamo condotto i pazienti guariti risultano essere l'83,3\% nel caso della tecnica chirurgica utilizzata nel gruppo A e l'82,1\% nei pazienti sottopo- 
sti a intervento con tecnica chirurgica più invasiva e quindi del gruppo B. La percentuale di recidive risulta essere del $16,6 \%$ e del $17,8 \%$, rispettivamente, nel gruppo A e B.

La percentuale di guarigione risulta coerente con quella emersa dagli studi precedenti, mentre non è stata riscontrata una differenza significativa per quanto riguarda le percentuali di recidive nei due diversi tipi di intervento. II fatto che la percentuale di recidiva sia di circa 1 caso su 5 , può essere spiegato sia considerando che i pazienti presi in esame in questo studio sono tutti onco-ematologici, quindi con un già presente deficit immunitario legato alla patologia che rende la guarigione della ferita meno facile, sia perché il numero ristretto del campione può non essere rappresentativo di una popolazione più grande. A questo bias può essere dovuta anche la mancata differenza tra le due tipologie di intervento. La possibilità di una recidiva è tanto maggiore quanto maggiore è la quantità di osso infetto e necrotico che viene lasciato in sede. Un intervento chirurgico resettivo tende a creare margini di osso netti più sani rispetto a un curettage che si avvale della sensazione e della vista dell'operatore. È vero anche che interventi più radicali talvolta - al fine di non deturpare troppo il viso del paziente, soprattutto nel mascellare superiore - tendono a essere sempre più conservativi, aprendo le porte al rischio di lasciare una piccola parte di osso da cui si scatena una nuova ONJ.

Nello studio che abbiamo svolto sono stati considerati unicamente pazienti onco-ematologici, escludendo pazienti che assumessero bifosfonati o denosumab per cause osteometaboliche. Inoltre, nonostante l'acronimo MRONJ comprenda anche la famiglia dei farmaci anti-angiogenetici, sono stati considerati unicamente i bifosfonati e il denosumab in modo da avere dei dati completi e il più affidabili possibili riguardo i pazienti consi- derati nello studio. Le principali complessità riscontrate nella ricerca sono state proprio la possibilità di avere accesso ai dati dei pazienti e, qualora questa fosse presente, la difficoltà nel trovare i dati utili e chiari riguardo la patologia di base e i farmaci assunti dal paziente. II numero finale dei pazienti reputati idonei allo studio risultano quindi essere solo una parte dei pazienti che hanno effettivamente sviluppato ONJ.

Essendo lo studio retrospettivo, gli interventi sono stati scelti sulla base della normale pratica clinica, quindi per lesioni estese-chirurgie invasive e per lesioni limitate di chirurgia conservativa. Potrebbe quindi essere normale che, nonostante le due tipologie di intervento siano diverse, non si siano ottenute differenze significative poiché le patologie di base erano già in partenza differenti. Per validare questa ipotesi sarà necessario disegnare uno studio clinico prospettico randomizzato controllato.

\section{CONCLUSIONI}

I risultati ottenuto da questo studio non dimostrano alcuna differenza statisticamente significativa tra i due approcci chirurgici nell'evitare le recidive in pazienti affetti da MRONJ (4 per il gruppo A vs 5 per il gruppo B), anche se, dato il campione ristretto, sarebbero necessari ulteriori studi, in particolare RCT, meglio se multicentrici, in maniera da evitare qualsiasi bias legato al numero esiguo di individui considerati.

\section{CONFLITTO DI INTERESSI}

Gli autori dichiarano di non avere alcun conflitto di interessi.

FINANZIAMENTI ALLO STUDIO

Gli autori dichiarano di non avere ricevuto nessun finanziamento per il presente studio.

CONSENSO INFORMATO

Gli autori dichiarano che è stato ottenuto il consenso informato del paziente per la pubblicazione del caso, foto incluse.

\section{BIBLIOGRAFIA}

1. Ruggiero SL, Dodson TB, Fantasia J, Goodday R, Aghaloo T et al. American Association of Oral and Maxillofacial Surgeons position paper on medication-related osteonecrosis of the jaw - 2014 update. J Oral Maxillofac Surg 2014;72(10):1938-56.
2. Di Fede O, Panzarella V, Mauceri R, Fusco V, Bedogni A et al. The dental management of patients at risk of medication-related osteonecrosis of the jaw: new paradigm of primary prevention. Biomed Res Int 2018;2018:2684924. 
3. Marx RE. Pamidronate (Aredia) and zoledronate (Zometa) induced avascular necrosis of the jaws: a growing epidemic. J Oral Maxillofac Surg 2003;61(9):1115-7.

4. Dodson TB. The frequency of medication-related osteonecrosis of the jaw and its associated risk factors. Oral Maxillofac Surg Clin North Am 2015;27(4):509-16.

5. Khan AA, Morrison A, Hanley DA, Felsenberg D, McCauley LK et al. Diagnosis and management of osteonecrosis of the jaw: a systematic review and international consensus. J Bone Miner Res 2015;30(1):3-23.

6. Christodoulou O, Pervena A, Klouvas G, Galani E, Falagas ME et al. Combination of bisphosphonates and antiangiogenic factors induces osteonecrosis of the jaw more frequently than bisphosphonates alone. Oncology 2009;76: 209-11.

7. Hellstein JW, Adler RA, Edwards B, Jacobsen PL, Kalmar JR, Koka S, Migliorati CA, Ristic H. Managing the care of patients receiving antiresorptive therapy for prevention and treatment of osteoporosis: executive summary of recommendations from the American Dental Association Council on Scientific Affairs. J Am Dent Assoc 2011;142(11):1243-51.

8. Graziani F, Vescovi P, Campisi G, Favia G, Gabriele M et al. Resective surgical approach shows a high performance in the management of advanced cases of bisphosphonate-related osteonecrosis of the jaws: a retrospective survey of 347 cases. J Oral Maxillofac Surg 2012;70(11):2501-7.
9. Kademani D, Koka S, Lacy MQ, Rajkumar SV et al. Primary surgical therapy for osteonecrosis of the jaw secondary to bisphosphonate therapy. Mayo Clin Proc 2006;81(8):1100-3.

10. Scoletta M, Arduino PG, Reggio L, Dalmasso P, Mozzati M et al. Effect of low-level laser irradiation on bisphosphonate-induced osteonecrosis of the jaws: preliminary results of a prospective study. Photomed Laser Surg 2010;28(2):179-84.

11. Vescovi $P$, Merigo E, Manfredi $M$, Meleti $M$, Fornaini $C$ et al. Nd:YAG laser biostimulation in the treatment of bisphosphonate-associated osteonecrosis of the jaw: clinical experience in 28 cases. Photomed Laser Surg 2008;26(1):37-46.

12. Ristow O, Ruckschloss T, Muller M, Berger M, Kargus S et al. Is the conservative non-surgical management of medication-related osteonecrosis of the jaw an appropriate treatment option for early stages? A long-term single-center cohort study. J Craniomaxillofac Surg 2019;47(3):491-9.

13. Bodem JP, Schaal, C, Kargus S, Saure D, Mertens C et al. Surgical management of bisphosphonate-related osteonecrosis of the jaw stages II and III. Oral Surg Oral Med Oral Pathol Oral Radiol 2016;121(4):367-72. 14. Comas-Calonge A, Figueiredo R, Gay-Escoda C. Surgical treatment vs. conservative treatment in intravenous bisphosphonate-related osteonecrosis of the jaws. Systematic review. J Clin Exp Dent 2017;9(2):e302-7.

15. Bedogni A, Saia G, Bettini G, Tronchet A, Totola A et al. Longterm outcomes of surgical resection of the jaws in cancer patients with bisphosphonate-related osteonecrosis. Oral Oncol 2011;47(5):420-4. 